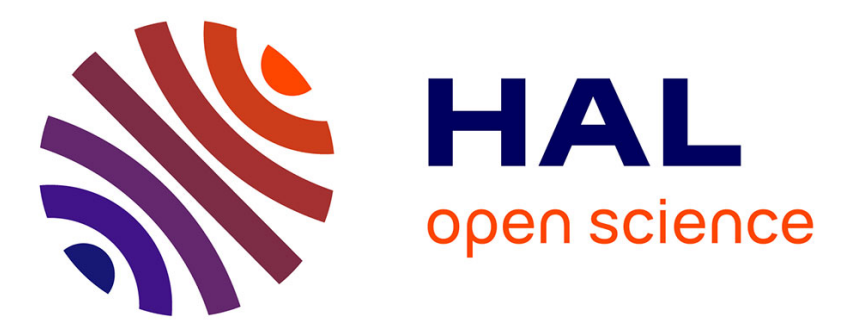

\title{
An end-member based ordering relation for the morphological description of hyperspectral images
}

Erchan Aptoula, Nicolas Courty, Sébastien Lefèvre

\section{To cite this version:}

Erchan Aptoula, Nicolas Courty, Sébastien Lefèvre. An end-member based ordering relation for the morphological description of hyperspectral images. IEEE International Conference on Image Processing (ICIP), Oct 2014, Paris, France. hal-00998256

\section{HAL Id: hal-00998256 https://hal.science/hal-00998256}

Submitted on 13 Nov 2019

HAL is a multi-disciplinary open access archive for the deposit and dissemination of scientific research documents, whether they are published or not. The documents may come from teaching and research institutions in France or abroad, or from public or private research centers.
L'archive ouverte pluridisciplinaire HAL, est destinée au dépôt et à la diffusion de documents scientifiques de niveau recherche, publiés ou non, émanant des établissements d'enseignement et de recherche français ou étrangers, des laboratoires publics ou privés. 


\title{
AN END-MEMBER BASED ORDERING RELATION FOR THE MORPHOLOGICAL DESCRIPTION OF HYPERSPECTRAL IMAGES
}

\author{
E. Aptoula \\ Okan University \\ Istanbul, 34959, Turkey
}

\author{
N. Courty, S. Lefèvre
}

Univ. Bretagne-Sud, UMR 6074 IRISA

Vannes, 56000, France

\begin{abstract}
Despite the popularity of mathematical morphology with remote sensing image analysis, its application to hyperspectral data remains problematic. The issue stems from the need to impose a complete lattice structure on the multi-dimensional pixel value space, that requires a vector ordering. In this article, we introduce such a supervised ordering relation, which conversely to its alternatives, has been designed to be imagespecific and exploits the spectral purity of pixels. The practical interest of the resulting multivariate morphological operators is validated through classification experiments where it achieves state-of-the-art performance.
\end{abstract}

Index Terms-Mathematical morphology, vector ordering, hyperspectral images, end-members, classification

\section{INTRODUCTION}

Mathematical morphology (MM) is one of the principal image analysis methods employed by the remote sensing community, especially in the context of filtering, segmentation and content description [1, 2, 3]. In addition to its rigorous theoretical foundation, its popularity has been further reinforced with the advent of sensors possessing higher spatial resolution, since it is inherently suitable for shape based analysis. Unfortunately however, MM has not been able to keep up with the advances in spectral resolution. Specifically, the exploitation of the information potential residing in hyperspectral images, that usually possess hundreds of bands, continues to be problematic, since MM cannot be straightforwardly extended to multivariate data.

In particular, the issue stems from the need to impose a complete lattice structure on the multi-dimensional pixel value space [4]. In fact, multiple attempts have been made to resolve this, most of which are variants of two basic strategies. The first, relies on effective dimension reduction (through e.g. PCA $[5,1]$ or ICA [6]), that removes inter-band redundancies and reduces hyperspectral images down to just a few bands. Following which, each image band is processed by means of existing grayscale morphological operators. Nevertheless, dimension reduction implies an inevitable data loss, while the independent processing of each band (a.k.a. marginal processing) multiplies the computational load, ignores any eventual inter-band correlation related information and can output vectors not included in the input image. That is why, a second strategy has emerged.

The alternative of marginal processing concerns developing a vector ordering relation for the high-dimensional pixels of hyperspectral images; thus avoiding dimension reduction and enabling the direct manipulation of hyperspectral image pixels. One of the earliest attempts in this context was made by [7], who employed cumulative spectral angular distances among vectors for determining their extrema, while [8] have employed the Euclidean norm of vectors for their comparison. More recently, [9] have introduced a supervised ordering, constructed through machine learning methods from two training pixel sets, one denoting arbitrarily the background and the other the foreground.

In this article, we follow the vector ordering paradigm, and introduce a novel ordering relation. Specifically, given the practical superiority of data-driven ordering methods $[10,11]$, we have chosen to develop an image specific approach. In detail, it orders vectors through their distances to automatically determined spectrally pure pixels. Hence, not only it does not require any arbitrarily chosen background and foreground pixel sets, but supports images with multiple spectral modes as well. This way, dilations lead to spectrally purer pixels while erosions lead to higher levels of mixing. The practical interest of the proposed approach is validated through classification experiments using a standard hyperspectral dataset, where it achieves state-of-the-art performance.

The rest of this article is organized as follows. Section 2 gives a detailed description of the proposed method, followed by experiments and the discussion of findings in Section 3; Section 4 concludes the paper.

\section{AN END-MEMBER BASED ORDERING}

\subsection{Definitions}

A complete lattice $\mathcal{L}$ is a non empty set equipped with a partial ordering $\leq$, such that every non-empty subset $\mathcal{P} \subset \mathcal{L}$ has both an infimum and a supremum. In this context, images 
are modeled by functions $f: \mathcal{E} \rightarrow \mathcal{T}$ mapping their domain space $\mathcal{E}$, usually $\mathbb{Z}^{2}$, onto a complete lattice $\mathcal{T}$, defining the set of possible "gray values".

Consequently, for a grayscale image $\mathcal{T}=\overline{\mathbb{Z}}$ or $\overline{\mathbb{R}}$, the underlying ordering used to impose a complete lattice structure on $\mathcal{T}$ is the usual comparison operator $\leq$. For a multichannel image $f$ on the other hand, $\mathcal{T}=\overline{\mathbb{Z}}^{n}$ or $\overline{\mathbb{R}}^{n}, n>1$, and $\mathcal{T}=\mathcal{T}_{1} \times \ldots \times \mathcal{T}_{n}$ is considered as the Cartesian product of $n$ complete lattices, where each mapping $f_{i}: \mathcal{E} \rightarrow \mathcal{T}_{i}, i \in$ $\{1, \ldots, n\}$ corresponds to an image band. Yet, the question of which ordering relation to employ in order to impose a complete lattice structure on the elements of $\mathcal{T}$ is in this case one with no universal answer. Once however such an ordering is established, the entire range of morphological operators can be directly implemented as mappings between complete lattices. For a comprehensive review of ordering methods the reader is referred to [4].

\subsection{End-members}

In the context of hyperspectral images, a pixel is a vector of reflectances sampled over different wavelengths. High spectral resolution usually leads to vectors of hundreds to thousand of values. At a given location, and because of the spatial resolution of the captor and scattering effects with various causes, the value contained in one pixel is assumed to be a combination of several spectra that describe the reflectance of a pure material (e.g. soil, water, asphalt, etc.). Those materials are said to be end-members, and as such constitute an idealized spectral response.

The determination of those end-members constitutes in itself a challenge for which various answers have been given in the last years [12]. We chose the classical N-FINDR algorithm [13] because of its simplicity. In a nutshell, the $\mathrm{N}$ FINDR starts first by reducing the data dimensionality, and then selects iteratively the end-members by choosing the element which maximizes the simplex formed by the previously chosen members and the new ones. Among the possible defects of the original form of N-FINDR, one can note its sensibility to the initial parameters, the a priori need for the number of end-members, and the assumption that the "true" endmembers are already available among the image pixels.

Usually, the computation of end-members enables the determination for each pixel of a proportion (i.e. abundance) of certain materials in the spectral signature. This is the classical unmixing problem [12], which is not the topic of this paper. In our approach, the end-members are used to devise a supervised ordering for the spectral data with the end of implementing morphological operators.

\subsection{Proposed ordering}

Grayscale images possess inherently minimum and maximum pixel values denoting respectively (but not necessarily) the background and foreground, and all values in-between are ordered w.r.t. their distances from those extrema. These notions however are absent from hyperspectral images. Of course, one can always pick arbitrarily two sets of pixels denoting the background and foreground and learn a vector ordering from them as suggested at [9]. Yet this strategy presumes that the input image is bi-modal, in the sense that it contains two basic pixel types or pixel classes. For example, sea and beach or forest and field. Thus one class can assume the role of the background and the other of the foreground. Yet, most often this is not the case with actual hyperspectral images, that usually possess several pixel classes, e.g. vegetation, buildings, shadow, water, etc.

Furthermore, considering that each end-member of a given hyperspectral image represents the spectrally purest member of each pixel class, we suggest exploiting them in order to establish an ordering relation that will take all of the pixel classes into account. At this point we borrow the idea of multiple reference based hue ordering presented in [14]. Specifically, given two vectors we can order them w.r.t. their distance to their corresponding closest end-member, with smaller distances denoting greater vectors. Consequently, dilations will result in images with pixel values closer to endmembers, thus spectrally purer, while erosions will lead to spectrally more mixed images.

Formally, let $\boldsymbol{f}$ be a hyperspectral image of $n$ bands $(n>>1)$ and $M=\left\{\boldsymbol{m}_{\boldsymbol{i}}\right\}_{1 \leq i \leq r}$ the set of $r$ end-members of $\boldsymbol{f}$, computed automatically through a method such as $\mathrm{N}$ FINDR. Thus, given a distance metric $d$ such as Euclidean, $\chi^{2}$ or spectral angular distance (SAD), one can order two vector pixels $\boldsymbol{f}(p)$ and $\boldsymbol{f}(q)$ as follows:

$$
\begin{aligned}
& \min _{i}\left\{d\left(\boldsymbol{f}(p), \boldsymbol{m}_{\boldsymbol{i}}\right)\right\}<\min _{j}\left\{d\left(\boldsymbol{f}(q), \boldsymbol{m}_{\boldsymbol{j}}\right)\right\} \\
& \Rightarrow \boldsymbol{f}(q)<_{M} \boldsymbol{f}(p)
\end{aligned}
$$

However, although it is highly improbable in practice for distinct vectors of high dimension to end up being equivalent w.r.t. Eq. (1) it still remains a possibility, and renders $<_{M}$ a pre-ordering, since it violates the anti-symmetry property. That is why we will equip this expression with a lexicographical comparison $\left(<_{l e x}\right)$ in order to avoid such pitfalls. Consequently, the end-member based ordering $\left(<_{M}\right)$ becomes:

$$
\begin{aligned}
& \boldsymbol{f}(q)<_{M} \boldsymbol{f}(p) \Leftrightarrow \\
& \left\{\begin{array}{c}
\min _{i}\left\{d\left(\boldsymbol{f}(p), \boldsymbol{m}_{\boldsymbol{i}}\right)\right\}<\min _{j}\left\{d\left(\boldsymbol{f}(q), \boldsymbol{m}_{\boldsymbol{j}}\right)\right\}, \text { or } \\
\min _{i}\left\{d\left(\boldsymbol{f}(p), \boldsymbol{m}_{\boldsymbol{i}}\right)\right\}=\min _{j}\left\{d\left(\boldsymbol{f}(q), \boldsymbol{m}_{\boldsymbol{j}}\right)\right\} \text { and } \\
\boldsymbol{f}(q)<_{\text {lex }} \boldsymbol{f}(p)
\end{array}\right.
\end{aligned}
$$

Naturally, considering that end-members are image specific, the resulting ordering relation will be so as well. All the same, given Eq. (2) one can define directly the basic morphological operators in the usual way [14].

Overall, Eq. (2) is an ordering method designed for dealing with hyperspectral images consisting of heterogeneous 
content (and not just bi-modal, in the sense of background and foreground). It does not require any intricate settings, except for the number of pixel classes present in the image, which can be easily set up in the context of supervised classification. Moreover, it could be straightforwardly modified in order to restrict comparisons only to certain end-members, thus leading to customized ordering relations, specific to the processing of certain materials; for example, in the case of marine target detection the end-members could correspond to the object of interest and to the sea, or in the case of geological exploration to the minerals of interest.

\section{EXPERIMENTS AND DISCUSSION}

The experimental validation of the presented ordering has been conducted through the classification of the Indian Pines hyperspectral dataset, where its performance is compared against three alternative ordering strategies. In detail, the dataset depicts an agricultural area with 16 distinct pixel classes, possesses 10, 249 labeled pixels, and has been acquired with the AVIRIS sensor (Fig. 1a). It is of size $145 \times 145$ pixels, containing 200 spectral bands at a $20 \mathrm{~m} /$ pixel spatial resolution.

In order to test our ordering relation, we have used the Euclidean distance in place of $d$ at Eq. (2), while the endmembers have been calculated using the N-FINDR algorithm explained in Section 2.2. The number of end-members to be computed has been set as equal to the number of class labels (i.e. 16).

The resulting ordering relation has been employed for implementing morphological profiles [15]. The feature vectors have been computed with disk shaped structuring elements of radius $3,5,7$ and 9 pixels in combination with multivariate openings and closings. Thus the profile of each band is of length 9, i.e. 4 values per filter, plus the original pixel value. Since the computation of profiles from each band would lead to excessively long feature vectors (e.g. $9 \times 200$-dimensional for Indian Pines), we have chosen to undertake this task by limiting the datasets to a smaller subset of their bands. And since N-FINDR requires at least $n$ bands in order to compute the same number of end-members, feature extraction has been conducted using 16 bands of the Indian Pines dataset (bands $\left.\{12 \times i\}_{1 \leq i \leq 16}\right)$. Thus the pixels are described by feature vectors of length $16 \times(4+1+4)=144$.

In addition to the proposed ordering (ENDM), the same feature vectors have been extracted using standard ordering strategies that have been already validated in this regard: marginal processing (MARG), the Euclidean norm based approach of [8] (NORM) and the cumulative extremum computation method of [7] employing spectral angular distances (SAD). The recently proposed method by [9] has been omitted from this stage, since it has been designed with bi-modal images in mind and requires the input of two pixel training sets, that are unclear how to select when dealing with the

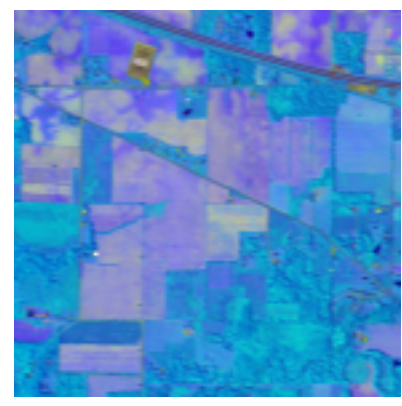

(a)

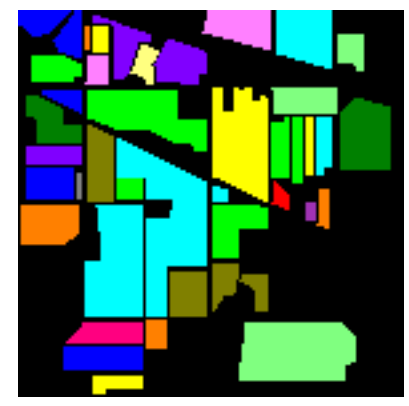

(b)
Fig. 1. The RGB color composite version and the training set pixels of the Indian Pines dataset.

classification of images containing tens of labels. Similarly, we have not involved the Extended Morphological Profile from [5] which requires a dimension reduction step through a PCA.

We used the classification setup of [1] and employed a Random Forest classifier composed of 100 trees, that were determined empirically to provide the best balance between learning speed and accuracy. The number of variables involved in the training of the classifier was set to the square root of the number of input variables. 10 pixels/class have been used for training while the remaining labeled pixels have been used for testing. The classification performance of each run has been measured in terms of average accuracy (AA) (i.e. average of class-wise accuracies) and overall accuracy (OA) (i.e. average of pixel-wise accuracies). Of course, for the sake of objectivity, the pixels of the training sets have been chosen randomly for each run, and the classification performances have been averaged across 30 runs.

Judging from the results shown in Table 1, ENDM slightly outperforms MARG, while both SAD and NORM are behind by several percentile points, in terms of both overall and average accuracy. The performance of SAD is in fact not a surprise as it does not constitute an ordering in the algebraic sense. Moreover, inter-class scores vary significantly, which could eventually be due to the varying detection quality of the end-members. If for instance the end-member of the class "corn-notill" has been detected erroneously, one cannot expect an effective description of those pixels using the proposed method. That is why, now that the theoretical groundwork is ready, future work will focus on exploring more effective end-member detection approaches.

\section{CONCLUSION}

This article focuses on the adaptation of morphological operators to hyperspectral images. They represent a harder challenge w.r.t. color images, due to both their high dimensionality and the less information availability on the relations between bands. In order to resolve the lack of ordering within 
Table 1. Classification accuracies (\%) for the Indian Pines image using 10 pixels per class for training. All values are averages across 30 runs using random training pixels.

\begin{tabular}{|c|c||c|c|c|c|}
\hline Class & Set Size & MARG (s.dev) & NORM (s.dev) & SAD (s.dev) & ENDM (s.dev) \\
\hline \hline Alfalfa & 46 & $94.99(1.74)$ & $91(1.71)$ & $97.87(1.74)$ & $\mathbf{9 7 . 2 1}(1.91)$ \\
\hline Corn-notill & 1,428 & $\mathbf{6 0 . 7 1}(1.85)$ & $56.69(1.49)$ & $39.68(1.87)$ & $59.5(1.69)$ \\
\hline Corn-mintill & 830 & $\mathbf{7 6 . 4 8}(1.63)$ & $70.13(1.23)$ & $43.05(1.63)$ & $73.54(1.54)$ \\
\hline Corn & 237 & $90.95(1.96)$ & $91.69(1.53)$ & $76.92(1.42)$ & $\mathbf{9 3 . 9 9}(1.62)$ \\
\hline Grass-pasture & 483 & $86.51(1.85)$ & $83.86(1.86)$ & $61.73(1.41)$ & $\mathbf{8 7 . 0 4}(1.87)$ \\
\hline Grass-trees & 730 & $89.8(1.84)$ & $85.34(1.67)$ & $78.08(1.31)$ & $\mathbf{9 1 . 8 8}(1.65)$ \\
\hline Grass-pasture-mowed & 28 & $99.77(1.45)$ & $\mathbf{1 0 0}(0.14)$ & $\mathbf{1 0 0}(0.11)$ & $\mathbf{1 0 0}(0.4)$ \\
\hline Hay-windrowed & 478 & $97.65(1.85)$ & $99.16(1.12)$ & $97.34(1.63)$ & $\mathbf{9 9 . 8 9}(1.9)$ \\
\hline Oats & 20 & $\mathbf{1 0 0}(0.2)$ & $96.4(0.34)$ & $94.8(1.62)$ & $\mathbf{1 0 0}(0.44)$ \\
\hline Soybean-notill & 972 & $70.53(1.01)$ & $66.19(1.45)$ & $53.8(1.41)$ & $\mathbf{7 2 . 5 7}(1.83)$ \\
\hline Soybean-mintill & 2,455 & $67.49(1.84)$ & $57.52(1.57)$ & $41.49(1.73)$ & $\mathbf{7 0 . 7 1}(1.95)$ \\
\hline Soybean-clean & 593 & $\mathbf{7 6 . 7 7}(1.9)$ & $72.55(1.15)$ & $47.23(1.92)$ & $76.01(1.84)$ \\
\hline Wheat & 205 & $97.99(1.85)$ & $97.59(1.46)$ & $96.66(1.28)$ & $\mathbf{9 8 . 4}(1.8)$ \\
\hline Woods & 1,265 & $\mathbf{9 3 . 1 1}(1.96)$ & $88.86(1.27)$ & $75.59(1.73)$ & $92.51(1.5)$ \\
\hline Build.-Grass-Trees-Drives & 386 & $94.95(1.84)$ & $84.15(1.83)$ & $80.5(1.84)$ & $\mathbf{9 5 . 4 3}(1.41)$ \\
\hline Stone-Steel-Towers & 93 & $\mathbf{9 9 . 0 4}(0.98)$ & $97.31(0.84)$ & $94.70(1.85)$ & $98.94(1.62)$ \\
\hline \hline Average Accuracy & & $87.3(1.65)$ & $83.65(1.29)$ & $73.72(1.53)$ & $\mathbf{8 7 . 9 8}(1.56)$ \\
\hline Overall Accuracy & & $77.76(1.95)$ & $72.27(1.55)$ & $57.36(1.74)$ & $\mathbf{7 8 . 5 8}(1.81)$ \\
\hline
\end{tabular}

the high-dimensional space of vector pixel values, we have proposed a new relation inspired from multiple reference based hue ordering. Specifically, our method orders vectors w.r.t. their spectral purity, through their distance to their closest end-member. It thus possesses a physical foundation conversely to its alternatives. In addition, with its current form the end-members are calculated only once per image and the resulting operators are image-specific. In the future, we could eventually obtain a more generic approach, by computing the end-members from a spectrally consistent set of hyperspectral images.

Besides exploiting the spectral purity of hyperspectral pixels in order to order them, our method additionally possesses a high level of flexibility, since one can limit its definition to the use of only certain end-members corresponding to materials of interest. Consequently one can obtain application specific ordering relations, such as the one of [9]. Its principal disadvantage on the other hand is the a priori requirement of the number of pixel classes, which is in fact a requirement of the N-FINDR method. Fortunately however, latest advances have enabled the automatic estimation of the number of end-members, thus opening the way for a fully parameterless ordering scheme.

The method introduced here has been tested in the context of image classification, where it has been used in order to implement morphological profiles, along with three alternative strategies. It has led to comparable scores w.r.t. the marginal ordering, despite of the latter's lack of vector preservation, since this property usually helps this approach to achieve better results. Future work will focus on the exploration of more sophisticated and less supervised end-member computation methods (that could eventually lead to an ordering relation practically superior to the marginal approach while being theoretically sounder), as well as alternative schemes for band selection and end-member distance computation. We finally intend to investigate the potential of our approach for developing application-specific ordering mechanisms, e.g. for hyperspectral object recognition.

\section{ACKNOWLEDGEMENTS}

This work was supported in part by the Scientific and Technological Research Council of Turkey (TUBITAK) under Career Grant 112E210, and by the French National Research Agency (ANR) under Project Grant ANR-13-JS02-0005-01 (Asterix project). This work has been realized during the corresponding author's stay at the Université de Bretagne Sud as an invited professor.

\section{REFERENCES}

[1] M. Dalla Mura, J. A. Benediktsson, B. Waske, and L. Bruzzone, "Morphological attribute profiles for the analysis of very high resolution images," IEEE Transactions on Geoscience and Remote Sensing, vol. 48, no. 10, pp. 3747-3762, 2010.

[2] M. Fauvel, J. A. Benediktsson, J. Chanussot, and J. R. Sveinsson, "Spectral and spatial classification of hyperspectral data using svms and morphological profiles," IEEE Transactions on Geoscience and Remote Sensing, vol. 46, no. 11, pp. 3804-3814, November 2008.

[3] Y. Tarabalka, J. Chanussot, and J. A. Benediktsson, "Segmentation and classification of hyperspectral images using watershed transformation," Pattern Recognition, vol. 43, no. 7, pp. 2367-2379, July 2010. 
[4] E. Aptoula and S. Lefèvre, "A comparative study on multivariate mathematical morphology," Pattern Recognition, vol. 40, no. 11, pp. 2914-2929, November 2007.

[5] J. A. Benediktsson, J. A. Palmason, and J. R. Sveinsson, "Classification of hyperspectral data from urban areas based on extended morphological profiles," IEEE Transactions on Geoscience and Remote Sensing, vol. 43, no. 3, pp. 480-491, March 2005.

[6] J. A. Palmason, J. A. Benediktsson, J. R. Sveinsson, and J. Chanussot, "Classification of hyperspectral data from urban areas using morphological preprocessing and independent component analysis," in IEEE International Geoscience and Remote Sensing Symposium, Seoul, Korea, July 2005, pp. 176-179.

[7] A. Plaza, P. Martinez, R. Pérez, and J. Plaza, "A new approach to mixed pixel classification of hyperspectral imagery based on extended morphological profiles," Pattern Recognition, vol. 37, no. 6, pp. 1097-1116, June 2004.

[8] P. Li and H. Hu, "Segmentation of high-resolution multispectral image based on extended morphological profiles," in Proceedings of the International Geoscience and Remote Sensing Symposium, Barcelona, Spain, July 2007, pp. 1481-1484.

[9] S. Velasco-Forero and J. Angulo, "Supervised ordering in $\mathbb{R}^{n}$ : Application to morphological processing of hyperspectral images,' IEEE Transactions on Image Processing, vol. 20, no. 11, pp. 3301-3308, 2011.

[10] E. Aptoula and S. Lefèvre, "Alpha-trimmed lexicographical extrema for pseudo morphological image analysis," Journal of Visual Communication and Image Representation, vol. 19, no. 3, pp. 165-174, April 2008.

[11] N. Courty, E. Aptoula, and S. Lefevre, "A classwise supervised ordering approach for morphology based hyperspectral image classification," in Proceedings of the International Conference on Pattern Recognition, Tsukuba, Japan, November 2012, pp. 1997-2000.

[12] J. M. Bioucas-Dias, A. Plaza, N. Dobigeon, M. Parente, Q. Du, P. Gader, and J. Chanussot, "Hyperspectral unmixing overview: Geometrical, statistical, and sparse regression-based approaches," IEEE Journal of Selected Topics in Applied Earth Observation \& Remote Sensing, vol. 5, no. 2, pp. 354-379, 2012.

[13] M. E. Winter, "N-FINDR: an algorithm for fast autonomous spectral end-member determination in hyperspectral data," in Imaging Spectrometry V, October 1999, vol. 3753 of Proceedings SPIE, pp. 266-275.
[14] E. Aptoula and S. Lefèvre, "On the morphological processing of hue," Image and Vision Computing, vol. 27, no. 9, pp. 1394-1401, August 2009.

[15] M. Pesaresi and J. Benediktsson, "A new approach for the morphological segmentation of high resolution satellite imagery," IEEE Transactions on Geoscience and Remote Sensing, vol. 39, no. 2, pp. 309-320, February 2001. 